\title{
Studying unquenching effects in QCD with Dyson-Schwinger equations *
}

Christian S. Fischer ${ }^{\mathrm{a}}$, Reinhard Alkofer ${ }^{\mathrm{b}}$, Wolfgang Cassing ${ }^{\mathrm{c}}$, Felipe Llanes-Estrada ${ }^{\mathrm{d}}$, Peter Watson ${ }^{\mathrm{e}}$, ${ }^{a}$ IPPP, University of Durham, Durham DH1 3LE, U.K.

${ }^{\mathrm{b}}$ Institute of Physics, Graz University, Universitätsplatz 5, A-8010 Graz, Austria

${ }^{\mathrm{c}}$ Institute for Theoretical Physics, Univ. of Giessen, Heinrich-Buff-Ring 16, 35392 Giessen, Germany

${ }^{\mathrm{d}}$ Fisica Teorica I, Univ. Complutense, Madrid 28040, Spain

${ }^{\mathrm{e}}$ Institute for Theoretical Physics, University of Tübingen, D-72076 Tübingen, Germany

We summarise recent results on the properties of gluons, quarks and light mesons from the Green's functions approach to QCD. We discuss a self-consistent, infrared power law solution for the Schwinger-Dyson equations of the 1PI-Greens functions of Yang-Mills theory. The corresponding running coupling has a universal fixed point at zero momentum. Based on these analytical results a truncation scheme for the coupled system of Schwinger-Dyson equations for the propagators of QCD and the Bethe-Salpeter equation for light mesons has been formulated. We compare numerical results for charge eigenstate vector and pseudoscalar meson observables with corresponding lattice data. The effects of unquenching the system are found to be small but not negligible.

One of the most fascinating problems of QCD is to find a low energy description of colourless bound states (hadrons) in terms of their nonperturbative, coloured constituents (quarks and gluons). Lattice simulations are not entirely satisfactory in this respect. They provide values for the global properties of hadrons (masses, decay widths etc.,), but they may not be capable to provide enough information on their internal structure to understand all dynamical aspects of low energy QCD. An alternative field theoretical and relativistic method which is well suited to deliver this information is the Schwinger-Dyson and Bethe-Salpeter formalism $[1,2]$. In principle this approach allows one to derive meson properties directly from the fundamental building blocks of the field theory, the Green's functions. Lattice simulations and the Green's functions approach are complementary to each other in several respects. Lattice simulations are ab initio whereas the Green's functions approach has to rely on an (educated) approximation scheme. The Green's

*Invited talk given by C. S. F. at the 'Workshop on computational hadron physics', Sept. 13 - 17, Nikosia, Cyprus. functions approach is continuum based. It allows for analytical investigations in the infrared and all aspects of chiral symmetry and its breaking pattern are respected. In this talk we summarise recent results in the Green's functions framework that dwell on these advantages. We discuss analytical results on the infrared exponents of the 1PI-Green's functions of SU $\left(N_{c}\right)$-Yang-Mills theory in Landau gauge. We report on numerical results for the ghost, gluon and quark propagators as well as light meson observables in a truncation scheme that is based on the analytical findings. Our focus in particular is on unquenching effects due to light quark loops in the gluon polarisation.

\section{Infrared exponents for the Green's func- tions of Yang-Mills theory}

The infrared behaviour of the Green's functions of $\mathrm{SU}\left(N_{c}\right)$-Yang-Mills theory is related to confinement in several ways. A particularly interesting example is the Kugo-Ojima confinement criterion of a well-defined global colour charge. The criterion is satisfied in Landau gauge if the dressing function of the ghost propagator is singular and 
the gluon propagator is finite or vanishes in the infrared. Provided BRST-symmetry is conserved nonperturbatively, the cohomology of the BRSToperator then defines a physical state space with colourless states only [3].

A convenient starting point to investigate the infrared behaviour of one-particle-irreducible (1PI) Green's functions is the Schwinger-Dyson equation (SDE) for the ghost-gluon vertex, shown diagrammatically fig. 1 . The dressed ghost-gluon vertex $\Gamma_{\mu}^{a b c}=\Gamma_{\mu}(p, q) f^{a b c}$ can be represented by the two tensor structures

$\Gamma_{\mu}(p, q)=p_{\mu} A\left(p^{2}, q^{2}\right)+k_{\mu} B\left(p^{2}, q^{2}\right)$,

where $p_{\mu}$ and $q_{\mu}$ are the momenta of the outgoing and incoming ghost and $k_{\mu}=-p_{\mu}-q_{\mu}$ is the gluon momentum. In Landau gauge, the momentum $q_{\mu}$ of the incoming ghost factorises from the vertex dressing, as can be seen from fig. 1: Since the gluon propagator $D_{\mu \nu}$ is transverse in Landau gauge, its contraction with the bare ghost-gluon vertex $l_{\mu}$ in the loop of the SDE gives $l_{\mu} D_{\mu \nu}(l-q)=q_{\mu} D_{\mu \nu}(l-q)$.

Let us assume for the moment that

(I) the loop-integral is finite in the infrared. (We come back to this assumption in the paragraph below eq. (6).) We then observe that the dressing of the full ghost-gluon vertex vanishes if $q_{\mu}$ goes to zero,

$\Gamma_{\mu}(p, q)=p_{\mu}(A-B)-q_{\mu} B \stackrel{q_{\mu} \rightarrow 0}{\longrightarrow} p_{\mu}$,

and thus neither $A\left(p^{2}, q^{2}\right)$ nor $B\left(p^{2}, q^{2}\right)$ can be singular in this limit [4]. Since factorisation of the other ghost momentum is trivial, the same happens for $p_{\mu} \rightarrow 0$. One thus concludes that the dressing of the ghost-gluon vertex is finite in the infrared and may be well approximated at small momenta by the bare vertex. This has been confirmed by lattice and SDE-studies [5-7].

A finite ghost-gluon vertex at small momenta admits the following power law solution for the ghost-SDE in the infrared: Writing the ghost and gluon propagators as

$$
\begin{aligned}
D^{G}\left(p^{2}\right) & =-\frac{G\left(p^{2}\right)}{p^{2}} \\
D_{\mu \nu}\left(p^{2}\right) & =\left(\delta_{\mu \nu}-\frac{p_{\mu} p_{\nu}}{p^{2}}\right) \frac{Z\left(p^{2}\right)}{p^{2}},
\end{aligned}
$$

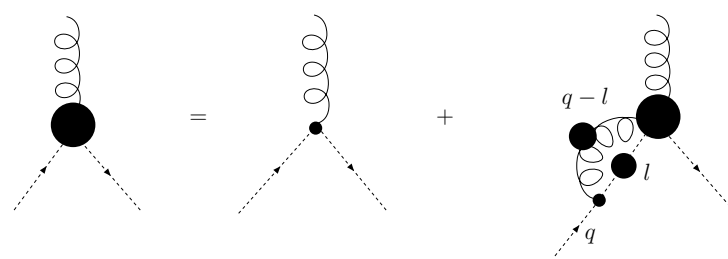

Figure 1. Schwinger-Dyson equation for the ghost-gluon vertex.

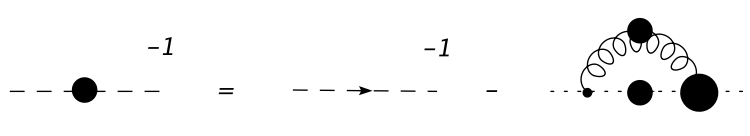

Figure 2. Schwinger-Dyson equation for the ghost propagator.

one finds power laws for the ghost and gluon dressing functions with interrelated exponents given by

$G\left(p^{2}\right) \sim\left(p^{2}\right)^{-\kappa}, \quad Z\left(p^{2}\right) \sim\left(p^{2}\right)^{2 \kappa}$.

[NB: This can be checked easily by just counting anomalous dimensions on both sides of the equation. Note that the loop-integral is dominated by momenta of the same magnitude as the external momentum. Thus, for small external momenta one can replace the propagators in the loop by their infrared approximation, eq. (4).] In this notation the Kugo-Ojima criterion translates to the condition $\kappa \geq 0$ for the ghost dressing function and $\kappa \geq 0.5$ for the gluon dressing function. On general grounds, the exponent $\kappa$ is known to be positive [4], independent of any truncation of the SDEs. For a bare ghost-gluon vertex in the infrared one obtains $\kappa=(93-\sqrt{1201}) / 98 \approx 0.595$ $[8,9]$, which satisfies both criteria. This specific value of $\kappa$ is found to vary only slightly for a large class of possible dressings of the ghost-gluonvertex [9]. Similar values have been determined from exact renormalisation group equations [10].

The basic idea to determine the infrared behaviour of the other (1PI) Green's functions is to 


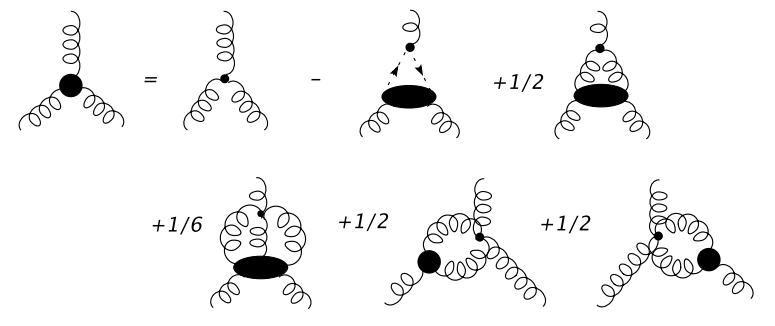

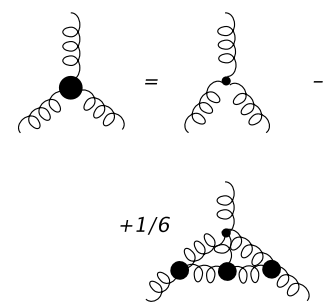

(c)

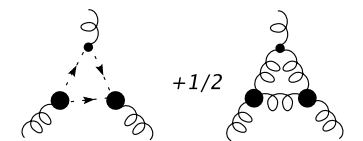

(b) (a)

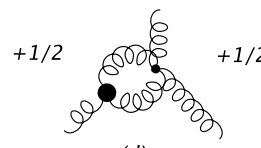

(d) (e)

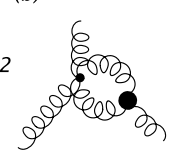

Figure 3. Exact Schwinger-Dyson equation for the three-gluon vertex and lowest order in a skeleton expansion of the four- and five-point functions. All internal propagators in the diagrams are to be understood as fully dressed.

investigate their Schwinger-Dyson equations order by order in a skeleton expansion (i.e. a loop expansion using full propagators and vertices). This program has been carried out in ref. [11]. It turns out that in this expansion the Green's functions can only be infrared singular, if all external scales go to zero. Thus to determine the degree of possible singularities it is sufficient to investigate the SDEs in the presence of only one external scale $p^{2} \ll \Lambda_{Q C D}^{2}$. As an example we summarise the treatment of the SDE for the three-gluon vertex. In fig. 3 we show the full equation as well as the lowest order in a skeleton expansion of the four-and five-point functions. In the presence of one (small) external scale the approximated SDE has a selfconsistent power law solution given by

$\Gamma^{3 g}\left(p^{2}\right) \sim\left(p^{2}\right)^{-3 \kappa}$.

[NB: Again this can be seen easily by counting anomalous dimensions on both sides of the equations. The leading diagram on the right hand side is the one involving ghosts, diagram (a), the others are less singular (recall $\kappa>0$ ). The loops are again dominated by momenta of the same magnitude as the external scale.] One can see by induction that this solution is also present if terms to arbitrary high order in the skeleton expansion are taken into account. Thus the skeleton expansion is stable wrt. the infrared solution of the SDEs. This technique can also be applied to any other SDE. A self-consistent solution of the whole tower of SDEs is then given by [11]

$\Gamma^{n, m}\left(p^{2}\right) \sim\left(p^{2}\right)^{(n-m) \kappa}$.

Here $\Gamma^{n, m}\left(p^{2}\right)$ denotes the infrared leading dressing function of the 1PI-Green's function with $2 n$ external ghost legs and $m$ external gluon legs. By counting anomalous dimensions it can be checked easily that the expression eq. (6) indeed solves the full three-gluon vertex SDE in fig. 3 selfconsistently. Furthermore, inserting $\Gamma^{1,2}\left(p^{2}\right) \sim\left(p^{2}\right)^{-\kappa}$ together with the power laws (4) into the SDE for the ghost-gluon vertex, fig. 1, one can verify the assumption (I) that the loop-integral of the vertex dressing is indeed finite in the infrared. Thus eq. (6) is a truly selfconsistent infrared solution of the tower of SDEs. [NB: It is worth mentioning that the solution (6) also has the correct scaling behaviour such that the Slavnov-Taylor identities of the renormalisation constants are satisfied. Since the theory is multiplicative renormalisable these functions scale with the renormalisation point $\mu^{2}$ in the same way as the $1 \mathrm{PI}-$ functions with the external scale $p^{2}$. E.g. the relation $Z_{1} / Z_{3}=\widetilde{Z}_{1} / \widetilde{Z}_{3}$ between the three-gluon vertex, gluon propagator, ghost-gluon vertex and ghost propagator renormalisation constant leads to $Z_{1}\left(\mu^{2}\right)=\left(\mu^{2}\right)^{-3 \kappa}$, which agrees with eq. (5).]

Certainly, selfconsistency is not enough to establish eq. (6) as the 'true' solution of Yang-Mills theory in the infrared, since there may be other selfconsistent solutions of the SDEs. However, one may argue that the solution (6) has an interesting property that qualifies it as a promising candidate: it leads to qualitative universality of the running coupling in the infrared. Renormalisation group invariant couplings can be defined from either of the primitively divergent vertices of Yang-Mills-theory, i.e. from the ghost-gluon 
vertex $(g h)$, the three-gluon vertex $(3 g)$ or the four-gluon vertex $(4 g)$ via

$$
\begin{aligned}
\alpha^{g h}\left(p^{2}\right) & =\frac{g^{2}}{4 \pi} G^{2}\left(p^{2}\right) Z\left(p^{2}\right) \quad \stackrel{p^{2} \rightarrow 0}{\sim} \frac{c_{1}}{N_{c}}, \\
\alpha^{3 g}\left(p^{2}\right) & =\frac{g^{2}}{4 \pi}\left[\Gamma^{0,3}\left(p^{2}\right)\right]^{2} Z^{3}\left(p^{2}\right) \stackrel{p^{2} \rightarrow 0}{\sim} \frac{c_{2}}{N_{c}}, \\
\alpha^{4 g}\left(p^{2}\right) & =\frac{g^{2}}{4 \pi}\left[\Gamma^{0,4}\left(p^{2}\right)\right]^{2} Z^{4}\left(p^{2}\right) \stackrel{p^{2} \rightarrow 0}{\sim} \frac{c_{3}}{N_{c}} .
\end{aligned}
$$

Using the SDE-solution (6) it is easy to see that all three couplings approach a fixed point in the infrared. The constants $c_{i}$ may be different for each coupling and depends on the respective choice of the tensor component used to extract the vertex dressing functions $\Gamma$ (this ambiguity is well know in the literature [12]). For the coupling (9) of the ghost-gluon vertex this fixed point can be explicitly calculated using propagator dressing functions alone. Employing a bare ghost-gluon vertex one obtains $\alpha^{g h}(0) \approx 8.92 / N_{c}$ [9]. Recently it has been shown that this value together with the infrared exponent $\kappa \approx 0.595$ are invariant in a class of transverse gauges that interpolate between Landau and Coulomb gauge [13].

\section{Unquenching effects in propagators}

Dynamical chiral symmetry breaking is, besides confinement, the most important low energy property of QCD. It is a truly nonperturbative effect, since there is no dynamical mass generation at every order in perturbation theory. On the quark level, the Schwinger-Dyson formalism is well suited to investigate the chiral symmetry breaking pattern also in the chiral limit of vanishing current quark masses. It is therefore complementary to lattice simulations, which provide reliable results for large quark masses and volumes, but are yet severely restricted close to the chiral limit. In the SDE-framework the effects of dynamical chiral symmetry breaking in quenched and (partially) unquenched QCD have been investigated in refs. $[14,15]$. Based on the analytical results summarised in the last section, ansätze for the vertices have been constructed such that the system of SDEs for the ghost, gluon and quark propagators are closed and can be solved numerically (for details of the truncation scheme see

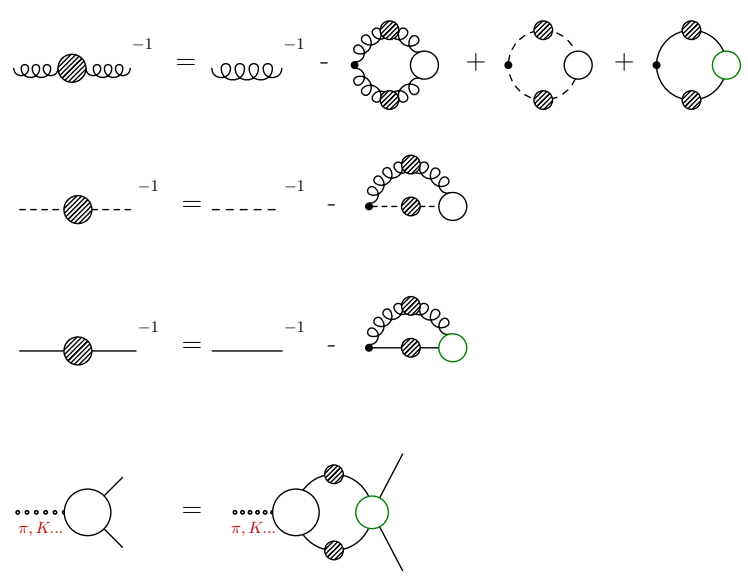

Figure 4. A diagrammatical representation of the coupled system of ghost, gluon and quark Schwinger-Dyson equations and the meson BetheSalpeter equation. Filled blobs denote dressed propagators and empty circles denote dressed vertex functions.

refs. $[14,16])$. Here we focus in particular on unquenching effects in these propagators, which are generated by quarks in the gluon SDE, cf. fig. 4 .

Numerical solutions for the ghost and gluon propagators can be seen in fig. 5. In the infrared, the numerical SDE-results reproduce the analytical power laws, eqs.(4). (This can be seen explicitly on a log-log-plot, displayed e.g. in Ref. [16].) In the ultraviolet they reproduce the correct oneloop running from resummed perturbation theory. Compared to the results of recent lattice calculations [17-19] (see also [20]) we find good agreement for large and small momenta. Small deviations for the value of the infrared exponent $\kappa$ between continuum-SDE and lattice results on a finite volume may have methodical reasons. The resulting running coupling on the lattice does not reproduce the fixed point behaviour in the continuum but vanishes in the infrared $[17,21,22]$. These effects are also seen when one solves Schwinger-Dyson equations on a torus and are discussed in detail elsewhere [23] (see 

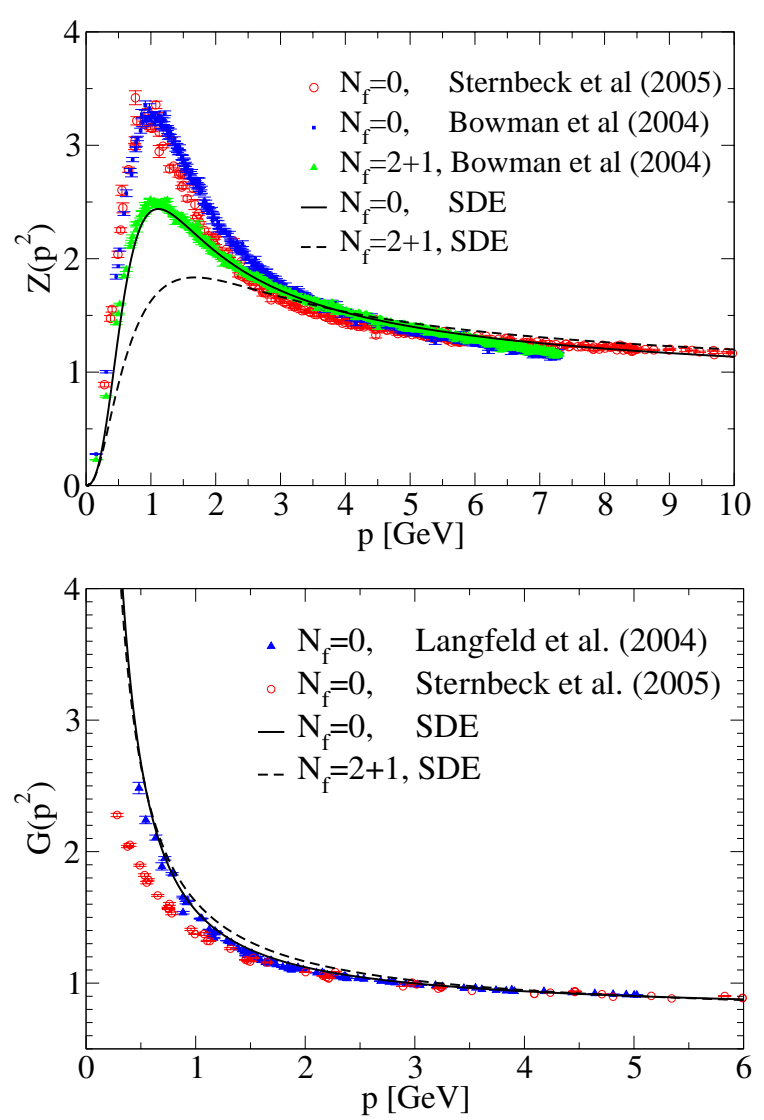

Figure 5. Comparison of the quenched and unquenched ghost and gluon dressing functions with recent lattice data [17-19]. The sea-quark masses are $m_{u / d} \simeq 16 \mathrm{MeV}, m_{s} \simeq 79 \mathrm{MeV}$ in the lattice simulations and $m_{u / d} \simeq 3.9 \mathrm{MeV}, m_{s} \simeq 84 \mathrm{MeV}$ in the SDE-approach.

also [24]). In the intermediate momentum region one clearly sees unquenching effects in the gluon dressing function due to the formation of quarkantiquark pairs from the vacuum. The screening effect from these pairs decreases the bump in the gluon dressing function considerably. The overall difference in the size of the bump between the lattice and the SDE-results is a measure of the influence of the (neglected) gluon-two-loop diagrams in the gluon-SDE.
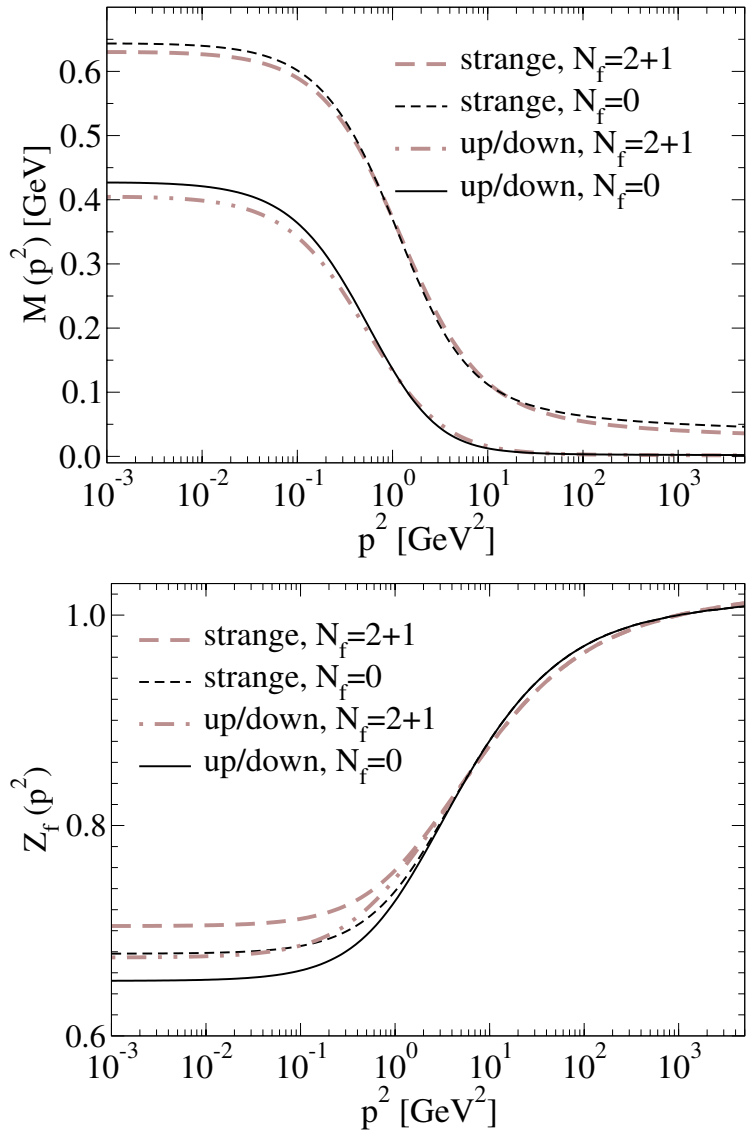

Figure 6. Comparison of the quenched and unquenched quark mass (upper diagram) and wavefunctions (lower diagram).

The up/down and strange quark propagator functions,

$S(p)=Z_{f}\left(p^{2}\right) /\left(-i \not p+M\left(p^{2}\right)\right)$

are plotted in fig. 6 . We clearly observe a large amount of dynamical mass generated in the infrared. This mass is reduced by roughly 10 percent once quark-loops are taken into account. For large momenta the numerical solutions reproduce the logarithmic running known from resummed perturbation theory. There are noticeable unquenching effects in the intermediate momentum region, which are, however, much smaller 
in size than those observed in the gluon propagator. By explicitly solving the quark-SDE in the complex plane one finds a pair of complex conjugate poles at $(0.47 \pm 0.29 \imath) \mathrm{GeV}$ (quenched) and $(0.45 \pm 0.27 \imath) \mathrm{GeV}$ (unquenched). Thus unquenching hardly has any effect on the position of these singularities (see however ref. [25] for a discussion of the possible influence of scalar tensor pieces in the quark-gluon vertex on the analytical structure of the propagator).

\section{Unquenching effects in light meson ob- servables}

The results for the gluon, ghost and quark propagators, discussed in the last section, serve as input for a calculation of light meson observables employing a Bethe-Salpeter equation (BSE), cf. fig. 4. The crucial link between the bound states and their quark and gluon constituents is provided by the axialvector Ward-Takahashi identity. It relates the quark self energy with the quark-quark interaction kernel in the BSE and thereby guarantees the Goldstone nature of the pions and kaons [26]. For details of the implementation of this identity within the truncation scheme discussed here see ref. [14]. Results for the masses of the pseudoscalar and vector meson (in the isospin symmetric limit) as a function of the current quark mass are shown in fig. 7. For $m_{q} \rightarrow 0$ (the chiral limit) we observe a massless pion as expected. As for unquenching effects both the pseudoscalar and vector masses with larger quark masses are increased $\sim 30 \mathrm{MeV}$ when quarks loops are taken into account.

The values for the current quark masses at the physical point together with the resulting pseudoscalar meson masses, leptonic decay constants and vector meson masses are given in Table 1 . When fitted to the experimental pion and kaon masses the resulting up/down and strange-quark masses are lowered when quark loop effects are taken into account. This has also been observed in corresponding lattice simulations $[27,28]$. We furthermore see that the results for $f_{K}$ and $m_{\rho}$ are quite insensitive to whether or not the system is unquenched (in the restricted sense of fig. 4). This leads to the conclusion that once the in-
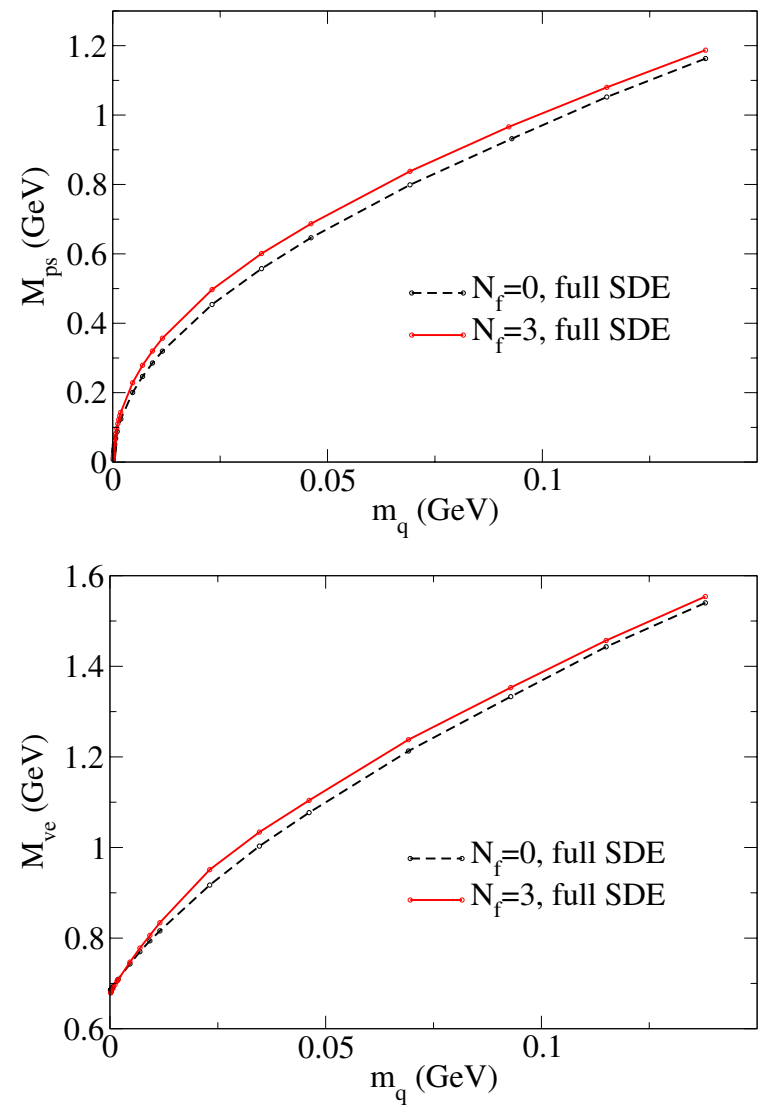

Figure 7. Pseudoscalar (upper diagram) and vector (lower diagram) meson masses as functions of the quark mass parameter. We compare results for the quenched and unquenched theory using three degenerate sea quarks.

teraction has been fitted to the pseudoscalar observables, the vector meson mass is largely fixed. The likely explanation for this is that the ground state pseudoscalar and vector mesons are both states with the lowest orbital angular momentum $(L=0)$ in the sense of the naive (quantum mechanical) quark model - meaning that they are determined largely by the lowest spin contributions of the kernel in the Bethe-Salpeter equation given by the ladder approximation (which is used here). The interaction then plays the same 


\begin{tabular}{|c|c|c|c||c|}
\hline$N_{f}$ & 0 & 3 & $2+1$ & PDG [29] \\
\hline \hline$m_{u}$ & 4.17 & 4.06 & 4.06 & $3-5$ \\
\hline$m_{s}$ & 88.2 & & 86.0 & $80-130$ \\
\hline$m_{\pi}$ & 139.7 & 139.7 & 140.0 & 139.6 \\
\hline$f_{\pi}$ & 130.9 & 131.1 & 131.0 & 130.7 \\
\hline$(-\langle\bar{q} q\rangle)^{1 / 3}$ & 266 & 271 & 271 & \\
\hline$m_{K}$ & 494.5 & & 493.3 & 493.7 \\
\hline$f_{K}$ & 165.6 & & 169.5 & 160.0 \\
\hline$m_{\rho}$ & 708.0 & 690.0 & 695.2 & 770.0 \\
\hline
\end{tabular}

Table 1

Parameter sets and results for $m_{\pi}, f_{\pi}, m_{K}, f_{K}$ and $m_{\rho}$ for the quenched case $\left(N_{f}=0\right)$, the unquenched case with three degenerate 'sea'-quarks $\left(N_{f}=3\right)$ and the physical quark configuration case $\left(N_{f}=2+1\right)$ with two up/down quarks and one strange quark. The quark masses and the condensate have been determined using a large renormalisation point and subsequently evolved down to the scale $\mu=2 \mathrm{GeV}$ according to their one-loop running. All units are $\mathrm{MeV}$.

role in both channels, hence the similarity in results. Note that the $\rho$ meson calculated within the framework of the truncated Bethe-Salpeter equation here refers to a pure quark-antiquark meson with no allowed decay channel. A first step towards including the non-trivial decay width of the physical $\rho$ meson in this formalism has been made in ref. [30] (see also ref [31] for a calculation of $g_{\pi \rho \rho}$ using quenched Bethe-Salpeter amplitudes). Here the decay of $\rho$ meson will lead to an additional shift of the $\rho$ meson mass peak as evident from dispersion relations. As a consequence our present results should not directly compared to experiment. Although the $\rho$ mass is slightly low the dispersive corrections via its two pion decay (and the inclusion of quark-gluon vertex corrections) might yield a satisfactory answer.

A suitable quantity to compare results from the Green's functions framework with lattice data is the vector meson mass as a function of the pseudoscalar meson mass. Since both quantities are physical no scheme ambiguities arise. The results are shown in fig. 8. From the SDE/BSE-approach we compare results from the full SDE-setup, de-

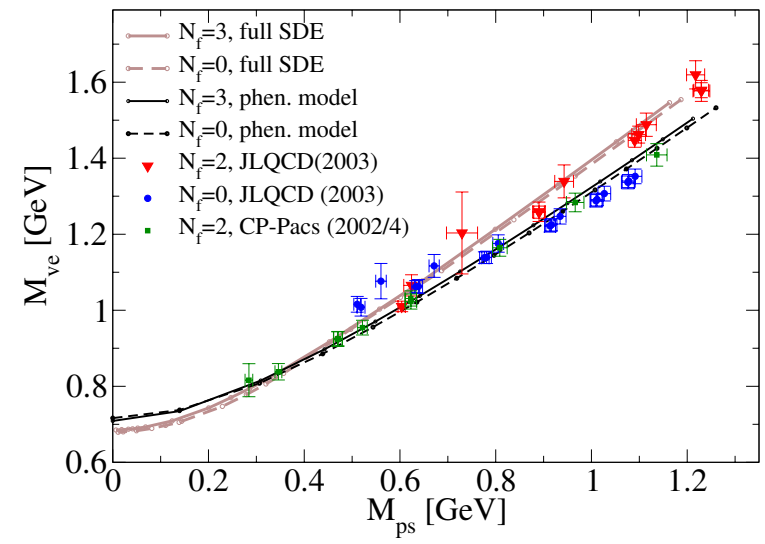

Figure 8. Vector meson masses as a function of pseudoscalar meson masses. We compare the quenched and degenerate unquenched cases. The lattice results are taken from refs. [27,28].

scribed above, with those obtained employing a phenomenological model for the quark-gluon interaction (the details of the model are described in ref. [14]). The model interaction and the effective interaction of the full SDE-setup are complementary to each other in the sense that the model interaction is confined to a quite narrow momentum region, whereas the interaction of the full SDE-setup has considerable strength in the infrared and extends into the ultraviolet according to the correct one-loop scaling known from perturbation theory. Together, both setups represent a measure for the theoretical error of our calculation. This error is obviously of the same size as the combined systematic error of the different lattice simulations. In general, the results are in nice agreement with the lattice data. For pion masses below $240 \mathrm{MeV}$, where no lattice data are available, the results show a nonlinear dependence of the vector meson mass on the pseudoscalar one. The effect of unquenching - when viewed as a function of the pseudoscalar meson mass - becomes the same for both schemes: the vector meson mass is slightly increased when quark loops are taken into account. This trend is also seen in the lattice simulations $[27,28]$, where the ef- 
fect is even more pronounced. However, these unquenching effects are small compared to the differences between both, the truncation schemes we employed and the systematic errors of the lattice results.

\section{Acknowledgements}

It is a pleasure to thank the organisers of the Workshop on computational hadron physics for all their efforts which made this highly interesting conference possible. C. S. Fischer and P. Watson thank M. Pennington for inspiring discussions. The work summarised here has been supported by a grant from the Ministry of Science, Research and the Arts of Baden-Württemberg (Az: 24-7532.23-19-18/1 and 24-7532.23-19-18/2), the Deutsche Forschungsgemeinschaft (DFG) under contract Fi 970/2-1, the Virtual Institute for Dense Hadronic Matter and QCD Phase Transitions and the Spanish FPA2005-02327.

\section{REFERENCES}

1. R. Alkofer and L. von Smekal, Phys. Rept. 353, 281 (2001).

2. P. Maris and C. D. Roberts, Int. J. Mod. Phys. E 12, 297 (2003).

3. N. Nakanishi and I. Ojima, World Sci. Lect. Notes Phys. 27, 1 (1990).

4. P. Watson and R. Alkofer, Phys. Rev. Lett. 86 (2001) 5239.

5. A. Cucchieri, T. Mendes and A. Mihara, JHEP 0412 (2004) 012.

6. A. Sternbeck et al., Proc. Sci. LAT2005, 333 (2005); these proceedings.

7. W. Schleifenbaum, A. Maas, J. Wambach and R. Alkofer, Phys. Rev. D 72, 014017 (2005).

8. D. Zwanziger, Phys. Rev. D 65, 094039 (2002).

9. C. Lerche and L. von Smekal, Phys. Rev. D 65, 125006 (2002).

10. J. M. Pawlowski, D. F. Litim, S. Nedelko and L. von Smekal, Phys. Rev. Lett. 93 (2004) 152002 ; C. S. Fischer and H. Gies, JHEP 0410 (2004) 048,

11. R. Alkofer, C. S. Fischer and F. J. LlanesEstrada, Phys. Lett. B 611, 279 (2005).

12. P. Pascual and R. Tarrach, Nucl. Phys. B $\mathbf{1 7 4}$ (1980) 123 [Erratum-ibid. B 181 (1981) 546].
13. C. S. Fischer and D. Zwanziger, Phys. Rev. D 72 (2005) 054005.

14. C. S. Fischer. P. Watson and W. Cassing, accepted for PRD, arXiv:hep-ph/0509213.

15. C. S. Fischer and R. Alkofer, Phys. Rev. D 67 (2003) 094020.

16. C. S. Fischer and R. Alkofer, Phys. Lett. B 536, 177 (2002); C. S. Fischer, R. Alkofer and H. Reinhardt, Phys. Rev. D 65, 094008 (2002).

17. A. Sternbeck, E. M. Ilgenfritz, M. MuellerPreussker and A. Schiller, Phys. Rev. D 72 (2005) 014507.

18. P. O. Bowman et al., Phys. Rev. D 70 (2004) 034509.

19. J. Gattnar, K. Langfeld and H. Reinhardt, Phys. Rev. Lett. 93, 061601 (2004).

20. O. Oliveira and P. J. Silva, AIP Conf. Proc. 756, 290 (2005).

21. B. Alles et al., Nucl. Phys. B 502 (1997) 325.

22. P. Boucaud et al., JHEP 0304, 005 (2003);

P. Boucaud et al., arXiv:hep-ph/0505150.

23. C. S. Fischer, B. Gruter and R. Alkofer, arXiv:hep-ph/0506053; C. S. Fischer, arXiv:hep-lat/0509031.

24. J. M. Pawlowski, D. F. Litim, S. Nedelko and L. von Smekal, AIP Conf. Proc. 756 (2005) 278.

25. R. Alkofer, W. Detmold, C. S. Fischer and P. Maris, Phys. Rev. D 70 (2004) 014014.

26. P. Maris, C. D. Roberts and P. C. Tandy, Phys. Lett. B 420 (1998) 267.

27. Y. Namekawa et al. [CP-PACS Collaboration], Phys. Rev. D 70 (2004) 074503. A. Ali Khan et al. [CP-PACS Collaboration], Phys. Rev. D 65 (2002) 054505 [Erratum-ibid. D 67 (2003) 059901].

28. S. Aoki et al. [JLQCD Collaboration], Phys. Rev. D 68 (2003) 054502.

29. S. Eidelman et al. [Particle Data Group], Phys. Lett. B 592 (2004) 1.

30. P. Watson and W. Cassing, Few Body Syst. 35 (2004) 99.

31. D. Jarecke, P. Maris and P. C. Tandy, Phys. Rev. C 67 (2003) 035202. 